\title{
Preparation of Polyanionic Cellulosic Microparticles with Antioxidant Capacity by Introducing Sulphurous Acid Groups onto Cellulose
}

\author{
Quan Ma, Xingzhong Zhang, Qianwen Shi, Yan Li, Bin Li, and Shilin Liu \\ College of Food Science \& Technology, Huazhong Agricultural University, Wuhan 430070, China \\ Correspondence should be addressed to Shilin Liu; slliu2013@mail.hzau.edu.cn
}

Received 5 June 2019; Accepted 9 July 2019; Published 24 July 2019

Guest Editor: Meng He

Copyright (c) 2019 Quan Ma et al. This is an open access article distributed under the Creative Commons Attribution License, which permits unrestricted use, distribution, and reproduction in any medium, provided the original work is properly cited.

\begin{abstract}
In this work, we presented a facile pathway to fabricate polyanionic cellulosic microparticles by selective oxidation with sodium periodate firstly, followed by grafting sodium bisulfite to the 2,3-dialdehyde cellulose particles. The obtained polyanionic cellulosic microparticles with the particle size of $2-4 \mu \mathrm{m}$ had increased stability in water, and it had $\mathrm{pH}$ responsiveness. Moreover, the polyanionic cellulosic microparticles had excellent film-forming property, and the tensile strength of the film formed from pristine cellulose particles was about $40 \mathrm{MPa}$, and it increased to $64 \mathrm{MPa}$ for the film formed from SRC-50 particles. Furthermore, the polyanionic cellulosic microparticles had certain reduction ability, and it could be used as coating to cover the surface of fruits or vegetables, and it could prevent the discoloration of the fresh-cut potato. The cellulose based particle coating with excellent antibrowning capacity, biocompatible, and environmentally friendly characteristics would be attractive for the applications in packaging material for food preservation.
\end{abstract}

\section{Introduction}

Coating is a powerful tool for modifying the surface properties of packaging materials [1-3]. It was well known that the surface properties have a key role in controlling the permeating of gas molecules, which could reduce the diffusion and migration rate of molecules in the film $[4,5]$. In the packaging field, coatings were either on the out surface or between two substrates formed sandwiched structure. Normally, the thickness of the coating layer ranged from tenths of nanometers to a few micrometers [6-8]. Currently, more and more interests were focusing on the possibility of using high performing biobased materials as functional layers to increase the sustainability of packaging materials $[9,10]$. Cellulose used in spray and foam coating technologies had some advantages; it could be used to coat very thin and uniform layers on the paper sheet, and some new functions like barrier properties for oxygen, water vapor, and grease/oil could be incorporated [11, 12]. Petri dishes modified with cellulose nanofibrils had some significant improvements in the mechanical properties, and the adding cellulose nanofibrils had a significantly improvement in air permeability. The improved mechanical strength and barrier properties of nanocellulose were related to its highly crystalline and cohesive energy density $[13,14]$. In brief, cellulose used as coating material had a great potential in producing novel packaging materials. This type of coatings was usually tasteless, bendy, and odorless and was of low energy, transparent, resistance to oil and fat, hydrophilic in nature, moderate to oxygen diffusion, and moisture [1517]. Moreover, it was one of the most promising candidates for using as biodegradable coating materials because of its biodegradability, abundance, and sustainability [18].

However, natural cellulose particle has poor stability in water and no active functional groups. In order to broaden the application fields of cellulose, functional modification was necessary [19-21]. Extensive researches had been performed to minimize the drawbacks of using coatings and making them more useful and functional in all ways. The in-situ synthesis of Ag nanoparticles in the cellulose matrix had been performed, and the Ag-cellulose composites had antimicrobial activity against E. coli and S. aureus [22]. Another 
pathway for the functionalization of the cellulose film with antibacterial properties was through dip-loading process; in this method, the cellulose film was immersed into the benzalkonium chloride solution; after being dried, the obtained cellulose film had antimicrobial activity against Staphylococcus aureus and Bacillus subtilis [23]. Paper coated with $\mathrm{CNF}$ and/or $\beta$-cyclodextrin $(\beta$-CD) with controlled release properties had also been developed to deliver antibacterial chlorhexidine digluconate [24]. Antioxidants, flavors, and color agents were also added in the cellulose coatings through different method, and some added functional components possessed greater film-forming function for the cellulose coating $[25,26]$.

We had put an intensive research on the cellulose dissolving and preparation of functional materials from the regenerated cellulose. In our previous works, a facile method had been developed for the preparation of regenerated cellulose particles with particle size less than $5 \mu \mathrm{m}$, and $\mathrm{O} / \mathrm{W}$ Pickering emulsion had been obtained by using the regenerated cellulose particles as stabilizers [27]. The effect of the contents of regenerated cellulose particles on the properties of the Pickering emulsions had been investigated. Furthermore, we presented a method for the hydrophobic modification of regenerated cellulose particles through selective oxidation with sodium periodate firstly, followed by grafting dodecylamine to the 2,3-dialdehyde cellulose particles, and the hydrophobic modified cellulose particles had increased emulsifiability in $\mathrm{O} / \mathrm{W}$ Pickering emulsion systems [28]. If the cellulose particle was used as coating materials, the stability in water was important for the application. Therefore, how to increase the stability of cellulose particles in aqueous state was a great challenge. In this work, polyanionic cellulosic microparticles had been prepared by selective oxidation with sodium periodate firstly, followed by grafting sodium bisulfite to the 2,3-dialdehyde cellulose particles. The polyanionic cellulosic microparticles had certain reduction ability, and it could be used as coating to prevent the discoloration of the fresh-cut potato that resulted from the oxidation. The cellulose based particle coating with excellent antioxidant capacity, biocompatible, and environmentally friendly characteristics would be attractive for the applications in packaging material for food preservation.

\section{Materials and Methods}

2.1. Materials. Cotton linters was purchased from Hubei Chemical Fiber Co., Ltd. Lithium hydroxide, urea, ethylene glycol, hydroxylamine hydrochloride, sodium bisulfite, sodium periodate, disodium hydrogen phosphate, sodium dihydrogen phosphate, trichloroacetic acid, potassium ferricyanide, and ferric chloride were purchased from Sinopharm Chemical Reagent Co., Ltd. Potassium bromide was purchased from Aladdin Chemical Reagent Co., Ltd. DPPH was purchased from Shanghai yuanye Bio-Technology Co., Ltd.

2.2. Preparation of Cellulose Gel Particles. LiOH/urea/water mixed solution was prepared by dissolving $8.70 \mathrm{~g}$ of $\mathrm{LiOH} \cdot \mathrm{H}_{2} \mathrm{O}$ and $12.00 \mathrm{~g}$ of urea in $79.30 \mathrm{~g}$ water. Then, 2.00 g cotton linter (native cellulose) was added into the mixed solution. The obtained cellulose suspension was put into refrigerator $\left(-20^{\circ} \mathrm{C}\right)$ for 12 hours. Then, it was thawed at room temperature to obtain cellulose solution. The cellulose solution was added into ethanol/water mixed solution with homogenization by using a homogenizer (IKA T18, Germany) at $5000 \mathrm{rpm}$; milk-like microparticles were obtained. After the removal of ethanol and inorganic salts, the cellulose gel particles were obtained.

2.3. Preparation of Sulfonated Regenerated Cellulose (SRC). For the preparation of the polyanionic cellulosic aqueous particles, there were mainly two steps named oxidation and grafting process for the chemical modification. Briefly, different amounts of sodium periodates were added into the flask with three necks containing cellulose particles $(100 \mathrm{~mL}$, $1.0 \mathrm{wt} \%)$. The weight of sodium periodates was controlled to $0.0132 \mathrm{~g}$ ( $10 \%$ in theoretical oxidation degree of cellulose), $0.0264 \mathrm{~g}$ ( $20 \%$ in theoretical oxidation degree of cellulose), $0.0396 \mathrm{~g}$ ( $30 \%$ in theoretical oxidation degree of cellulose), $0.0528 \mathrm{~g}$ ( $40 \%$ in theoretical oxidation degree of cellulose), and $0.066 \mathrm{~g}$ ( $50 \%$ in theoretical oxidation degree of cellulose) according to the theoretical oxidation degree of cellulose, respectively. The reaction was carried out at $38^{\circ} \mathrm{C}$ for $6 \mathrm{~h}$. The obtained oxidized cellulose particles based on the theoretical oxidation degree were coded as ORC-10, ORC-20, ORC-30, ORC-40, and ORC-50, respectively. Afterwards, the $\mathrm{pH}$ was adjusted to 4.5 for the following grafting reaction. $\mathrm{NaHSO}_{3}$ was added into the oxidized cellulose particles with stirring. The reaction was carried out at $38^{\circ} \mathrm{C}$ for $5 \mathrm{~h}$ under stirring. Then the sample was dialyzed with deionized water and then freeze-dried. The obtained grafted cellulose particles were coded as SRC-10, SRC-20, SRC-30, SRC-40, and SRC-50, respectively. The pristine regenerated cellulose gel dispersion was coded as RCG.

2.4. Determination of Cellulose Oxidation Degree. The oxidation degree (OD) of the cellulose films was determined according to the reported method based on the Schiff base reaction between aldehyde groups and hydroxylamine hydrochloride. The oxidation degree was calculated through the following equation [29]:

$$
O D=\frac{161 \times\left(v_{2}-v_{1}\right)}{m} \times c(H C l) \times 10^{-3}
$$

where $v_{2}$ was the volume of hydrochloric acid consumed $(\mathrm{mL}) ; v_{1}$ was the volume of hydrochloric acid consumed in blank experiment $(\mathrm{mL}) ; c_{(\mathrm{HCl})}$ was the concentration of hydrochloric acid; $m$ was the weight of each sample; and 161 was the average molecular weight when glucose units were translated into dialdehyde.

2.5. Determination of Reduction Capacity of SRC. The scavenging activity of hydroxyl radicals was measured according to the reported method [30]. $2.0 \mathrm{~mL}$ test sample was mixed with $2.0 \mathrm{~mL}$ of phosphate buffer $(0.2 \mathrm{mM}, \mathrm{pH} 6.6)$ and 2.0 $\mathrm{mL}$ of $1 \%$ potassium ferricyanide $(\mathrm{w} / \mathrm{v})$. The mixtures were incubated for $20 \mathrm{mins}$ at $50^{\circ} \mathrm{C}$. After the incubation, $2.0 \mathrm{~mL}$ of 


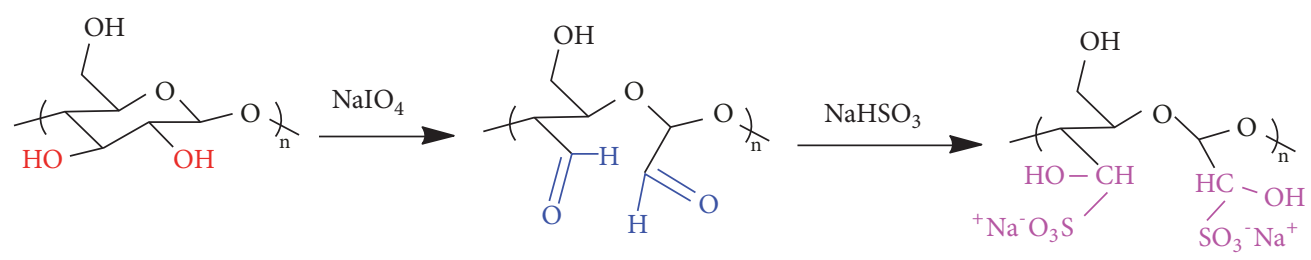

FIGURE 1: The chemical process for the introducing sulphurous acid groups onto cellulose through selectively oxidization and addition reaction.

$10 \%$ trichloroacetic acid was added to the mixtures, followed through centrifugation at $3000 \mathrm{rpm}$ for 10 mins. The upper layer $(2 \mathrm{~mL})$ was mixed with $2 \mathrm{~mL}$ of distilled water and 0.4 $\mathrm{mL}$ of $0.1 \%$ ferric chloride. The reaction mixture was shaken and incubated for 10 mins, and the absorbance of the resultant solution was measured at wavelength of $700 \mathrm{~nm}$.

2.6. Characterizations. Fourier transform infrared (FT-IR) tests were carried out with a FT-IR analyzer (470-Nexus, Nicolet, USA) in wavenumbers ranging from 400 to 4,000 $\mathrm{cm}^{-1}$. The samples were ground into powder and pressed with $\mathrm{KBr}$ to form a transparent pellet for the test. The morphology of cellulose particles was characterized by using a microscope equipped with a digital camera (Nikon 80i, Japan). The zeta potential of the pristine and modified cellulose particles was characterized with a Malvern Zetasizer Nano ZEN 3600. The rheological behavior of the cellulose microparticles suspensions and the formed emulsions were investigated by using Discovery HR-2 Hybrid Rheometer (TA Instruments, New Castle, DE, USA). Steady shear flow tests were performed at shear rates ranging from 1 to $1000 \mathrm{~s}^{-1}$. The test temperature was kept at $25 \pm 0.1^{\circ} \mathrm{C}$; a layer of silicon oil was used to cover the exposed surface of the suspension to avoid the dehydration during the test process. The mechanical properties of the films were characterized with a tensile tester (CMT 6503, Shenzhen SANS Test machine Co. Ltd., China) according to ASTM/D638-91 with a speed of $2 \mathrm{~mm} \bullet \mathrm{min}^{-1}$.

Statistics. Statistical analyses were carried out by using the SPSS 13.0 statistical analysis program, and the differences between trials were detected by Tukey test $(\mathrm{P}<0.05)$. The results reported in the manuscript were for at least 3 times of repeated tests.

\section{Results and Discussion}

Surface-modified cellulose gel particles could be fabricated by combining oxidation reaction and grafting reaction to improve properties and expand applications of cellulosic materials. The chemical process for the introducing sulphurous acid groups onto cellulose through selectively oxidization and addition reaction was shown in Figure 1. Periodate $\left(\mathrm{NaIO}_{4}\right)$ was used to oxidize the 2,3- hydroxyl groups in glucose unit; the obtained 2, 3-dialdehyde cellulose (DAC) was further introduced sulphurous acid groups via the addition reaction with sodium bisulfite $\left(\mathrm{NaHSO}_{3}\right)$. With the increase of the concentration of $\mathrm{NaIO}_{4}$, the aldehyde content

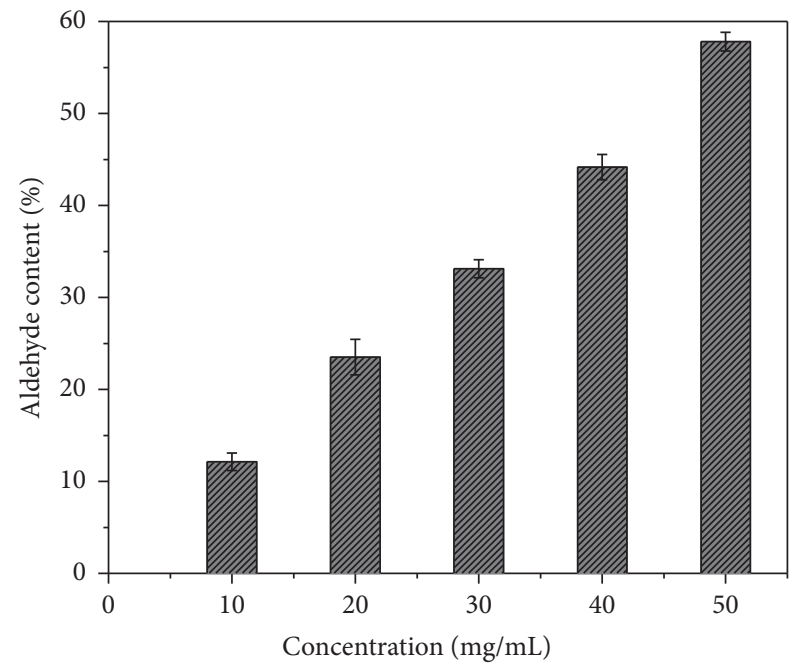

FIgURE 2: The influence of the concentration of $\mathrm{NaIO}_{4}$ on the generated content of aldehyde groups in the cellulose dispersions.

of ORC was increased from $12.13 \%$ to $57.8 \%$, as it was shown in Figure 2. It indicated that more sulphurous acid groups could be grafted onto cellulose with the increased content of aldehyde groups, which would increase the stability of the cellulose particles in water, for more charged groups were introduced into cellulose scaffolds.

The dispersed state of the cellulose particles and sulfonated regenerated cellulose particles (SRCs) in water was shown in Figure 3. The SRC microparticles were welldispersed, and the optical transmittance of dispersions was higher than that of the cellulose particles dispersions. The clearness of the SRCs was increased with the increasing degree of oxidation. The zeta potential of the regenerated cellulose particles was about $-14 \mathrm{mV}$ under neutral condition, and it was decreased to $-23 \mathrm{mV}$ for SRC-30, and it could be further decreased to about $-54 \mathrm{mV}$ for SRC-50. It indicated that the stability and dispersion of SRCs were improved. The high zeta potential of SRCs dispersions was ascribed to the contribution of sulphurous acid groups.

The morphologies of the RCG and SRCs particles were shown in Figure 4. The particle size of the RCG was about $5 \mu \mathrm{m}$, as it was shown in Figure 4(a). While for the sulphurous acid modified cellulose microparticles, the particle size of SRCs was decreased to $2-4 \mu \mathrm{m}$ after the chemical modification process, and the stability of the modified cellulose microparticles had been improved. It suggested that 


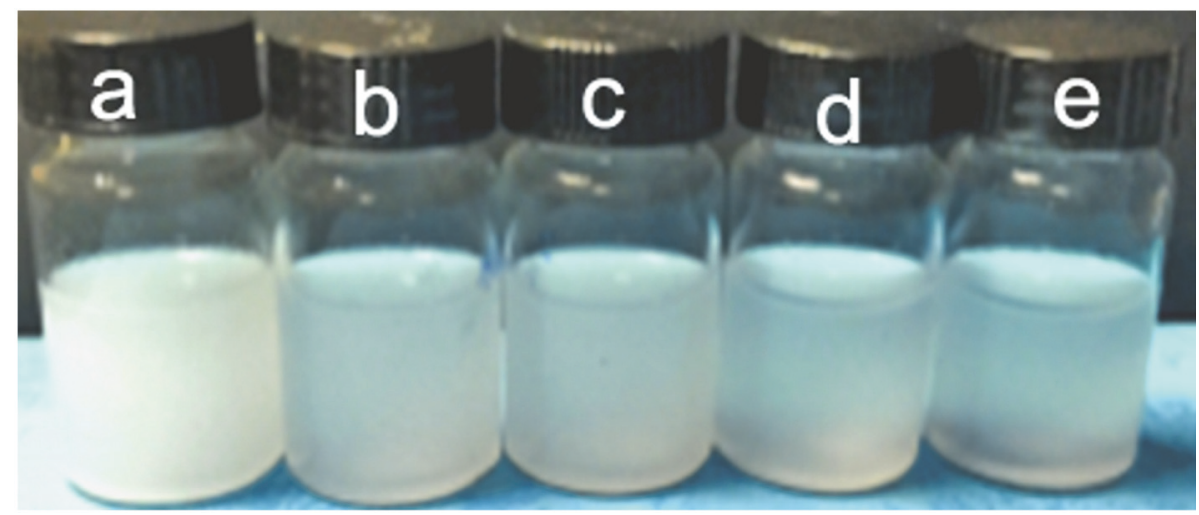

FIgURE 3: Photos of the RC dispersions (a) and SRC with different contents of sulphurous acid groups; (b)-(e) were for SRC-10, SRC-30, SRC-40, SRC-50, respectively.

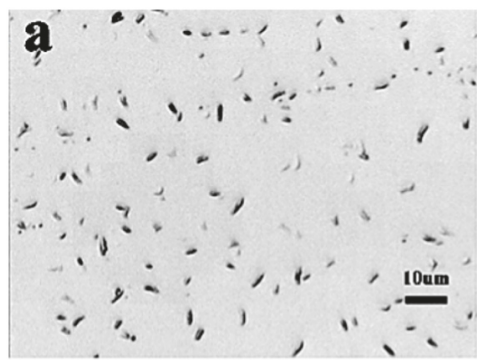

(a)

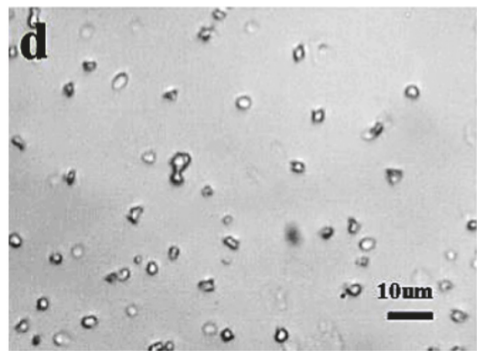

(d)

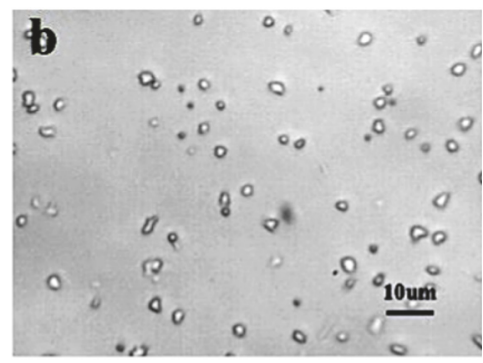

(b)

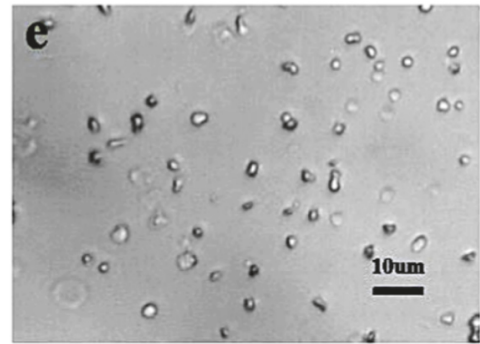

(e)

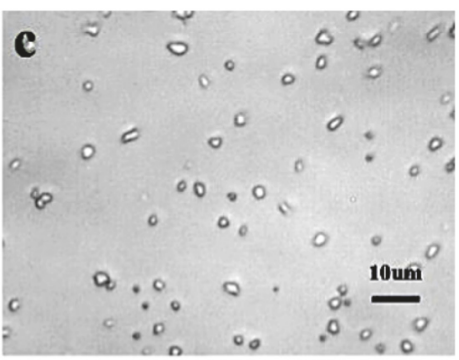

(c)

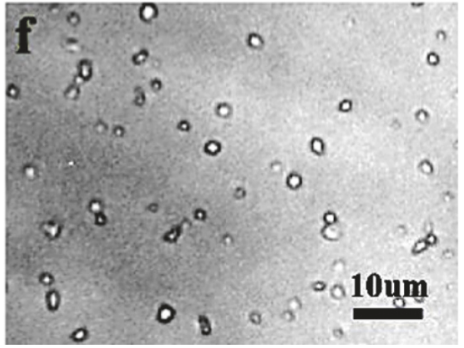

(f)

FiguRE 4: Optical microscopy images of the cellulose gel particles (a) prepared from cellulose solution with concentration of 2.0 wt\%; (b)-(f) were for SRC-10, SRC-20, SRC-30, SRC-40, SRC-50, respectively.

the appropriate hydrophilic modification of the surface of the cellulose particles could increase its dispersion stability. Figure 5 showed the FT-IR spectra of the RC, ORC, and SRC. All samples had the absorption peaks of $1100 \mathrm{~cm}^{-1}$ and 1380 $\mathrm{cm}^{-1}$, which corresponded to the $\mathrm{C}-\mathrm{O}-\mathrm{C}$ asymmetric stretching and $-\mathrm{CH}_{2}$ bending vibration of cellulose, respectively. The absorption peaks of $-\mathrm{C}=\mathrm{O}$ stretching appeared at $1726 \mathrm{~cm}^{-1}$ in ORC, and the absorption peaks of hydroxyl group $\left(1159 \mathrm{~cm}^{-1}\right.$ and $1059 \mathrm{~cm}^{-1}$ ) were observed in SRC; it indicated that the reaction of oxidation and grafting were successfully carried out on RCG particles. Meanwhile, the content of aldehyde group was increased with the increase of the concentration of $\mathrm{NaIO}_{4}$, according to the enhanced stretching vibration peaks of aldehyde group. While the absorption peaks of $-\mathrm{C}=\mathrm{O}$ on
SRC was almost disappeared, which suggested that the sulfite characteristic groups $(-\mathrm{S}=\mathrm{O})$ were effectively immobilized onto the ORC. The zeta potential and aggregation of SRC particles was tended to be affected by $\mathrm{pH}$. Therefore, the influence of $\mathrm{pH}$ on the stability of obtained SRC was characterized. With the increasing of $\mathrm{pH}$ from 2.0 to 10.0 , the zeta potential was increased first and then decreased, and the SRC particles had the maximum potential absolute value at $\mathrm{pH}$ 5.0. When the $\mathrm{pH}$ was lower than 4.0 , the zeta potential absolute value of SRC particles was below $20 \mathrm{mV}$. The gel particles were unstable and tended to flocculate. When the $\mathrm{pH}$ was exceeded 8.0, the particles became unstable with the decreasing potential values. The stability of the SRC particles in different $\mathrm{pH}$ could present the information of its application conditions. 


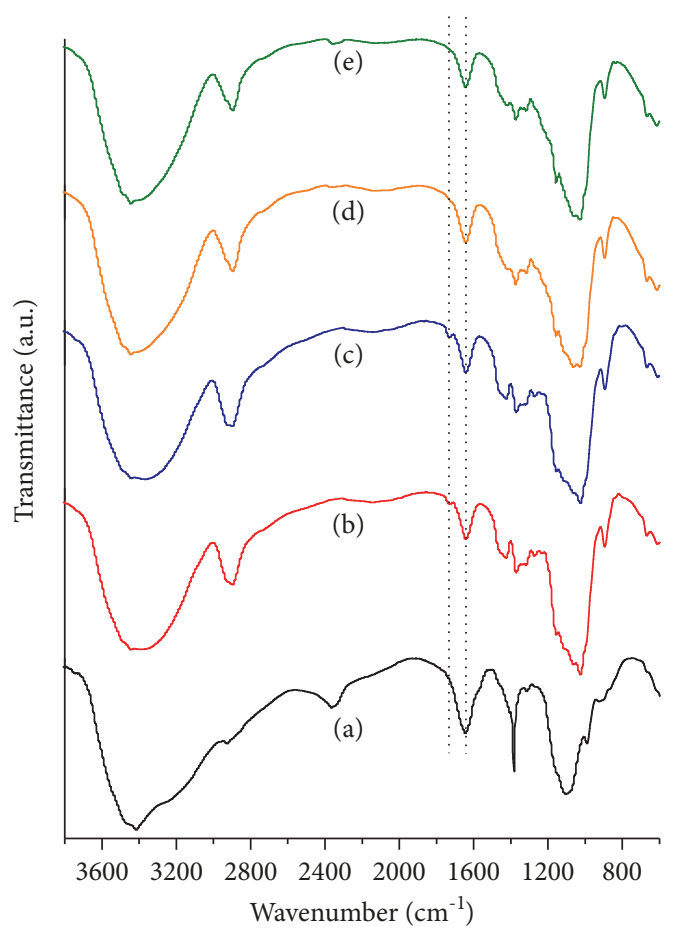

Figure 5: FT-IR spectra of (a) RC dispersions, (b) ORC-10, (c) ORC30, (d) SRC-10, and (e) SRC-30, respectively.

Figure 6 showed the stress-strain curves of the films prepared from RCG and SRC with different oxidation degrees at ambient conditions. The stress was gradually increased, and the stress of SRC films was higher than that of pure RC film. The stress and strain points at break for RC, SRC-20, SRC-30, SRC-40, and SRC-50 films were measured as $39.9 \mathrm{Mpa}, 3.5 \%$; 57.3 Mpa, 1.7\%; 43.4 Mpa, 1.1\%; 62.6 Mpa, 2.5\%; and 64.1 Mpa, $0.8 \%$, respectively. It was obvious that the tensile strength of the SRC was higher than that of the RC films. It indicated that the mechanical property of the SRC films was not destroyed through the oxidation process of cellulose for the grafting reaction. The increased mechanical strength of SRC films was important for their potential application as coating materials.

The grafting of sulfite ions $\left(\mathrm{SO}_{3}{ }^{2-}\right)$ could endure materials with strong reducibility, which could effectively inhibit the oxidation and brown of fruits and vegetables. Therefore, reducing power assay was used to evaluate the potential antioxidant activity of RC and SRC with different oxidation and grafting degrees. As shown in Figure 7, the absorbance of SRC obviously increased with the increasing concentrations, while the RC had little change in the absorbance; it suggested that unmodified RC gel particles had no antioxidant activity. At a concentration of $5 \mathrm{mg} / \mathrm{mL}$, the absorbance of SRC-20, SRC-30, SRC-40, and SRC-50 was about 0.554, 0.616, 0.746, and 0.925 , respectively. The results showed that the reducing power increased with the increasing content of sulfite ions grafted on the surface of cellulose, which indicated that SRC gel particles had enhanced antioxidant activity, and the antioxidant activity was increased with increasing the solid contents of the SRC particles.

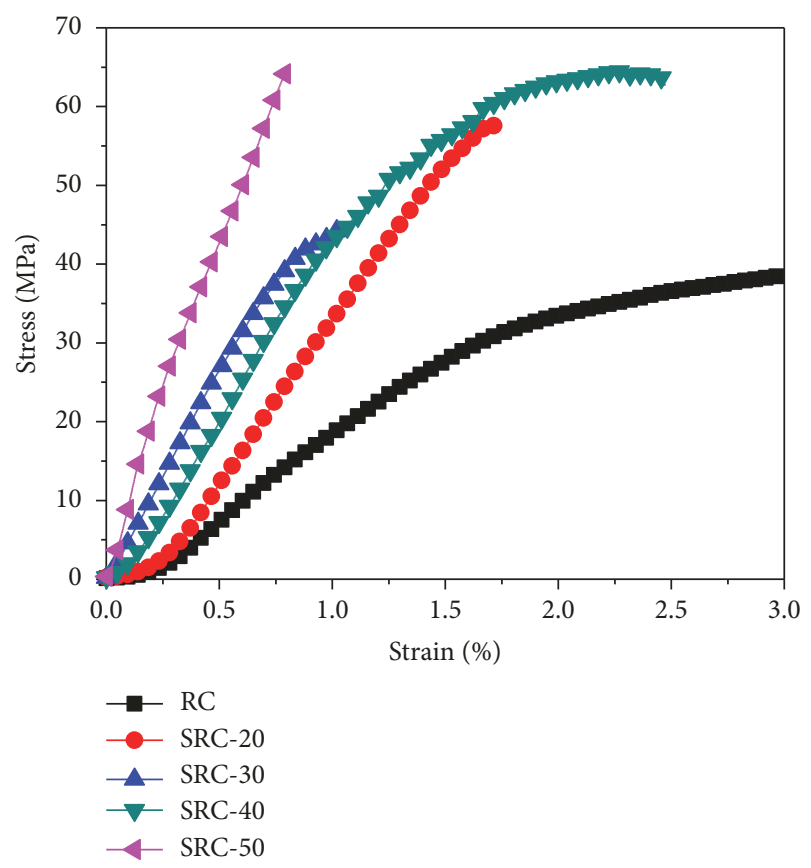

FIGURE 6: The stress-strain curves of the films prepared with RC dispersion and SRCs; the solid content of the dispersions for the preparation of the films was control to $1.2 \mathrm{wt} \%$.

In addition, the SRC gel particles with good film-forming property and antioxidant activity could be a promising antibrowning agent for fresh-cut fruits or vegetables. The color changes of potato slices treated with SRC dispersions were measured by a colorimeter. The $\Delta E$ was an indicator of integrated color differences comparing to the color of initial time, calculated as [31]

$$
\Delta \mathrm{E}=\sqrt{\Delta L^{* 2}+\Delta a^{* 2}+\Delta b^{* 2}}
$$

where $L^{*}$ was the lightness, $a^{*}$ was the reddish-greenish, and $b^{*}$ was the yellowish-bluish. The $L^{*}$ had a high correlation with the potato slices browning, which decreased with the increasing degree of browning discoloration. In Figure 8, the value of $L^{*}$ gradually decreased but the value of $\Delta \mathrm{E}$ gradually increased with storage time. Importantly, the photo slices treated with SRC-50 dispersions had the highest $\mathrm{L}^{*}$ value and the lowest $\Delta E$ value at all the time points, which confirmed that the SRC-50 gel particles had an excellent antibrowning capacity.

\section{Conclusions}

In this work, polyanionic cellulosic microparticles with antioxidant capacity by introducing sulphurous acid groups onto cellulose were successfully prepared by combining oxidation and grafting reaction. The obtained SRC dispersions were well-dispersed and expressed the regular shape of sheet or bulk with the particle size of $2-4 \mu \mathrm{m}$ by micromorphology observation. The formation of aldehyde group after oxidation was verified by FT-IR spectra, and the aldehyde content of ORC increased from $12.13 \%$ to $57.8 \%$ with the increase of 


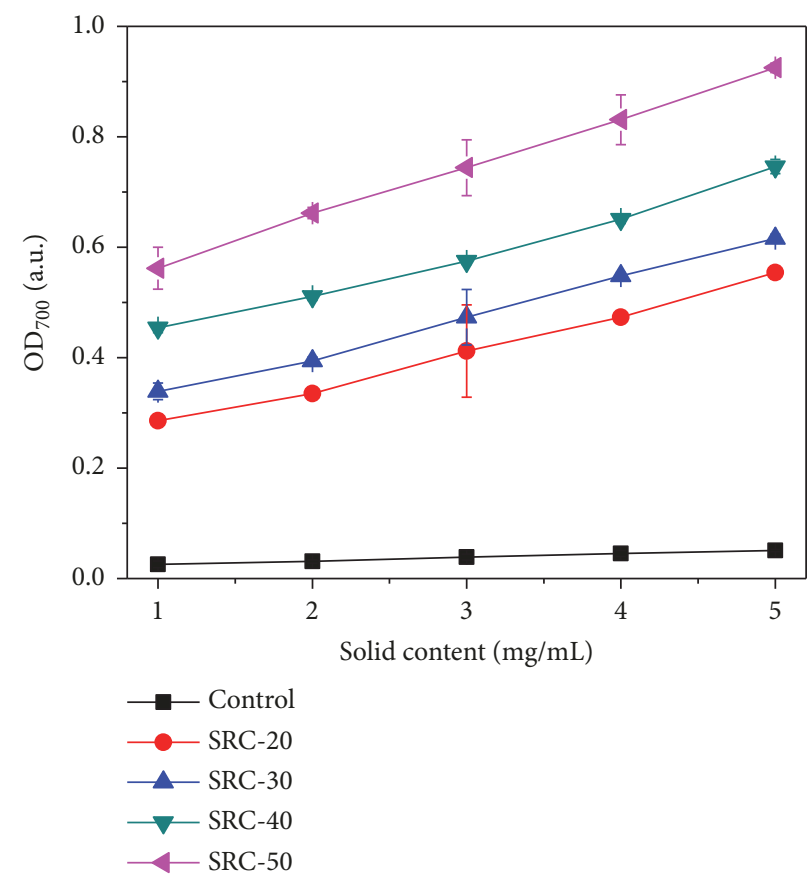

FIGURE 7: The relationship between the content of the SRCs and the reduction capacity.

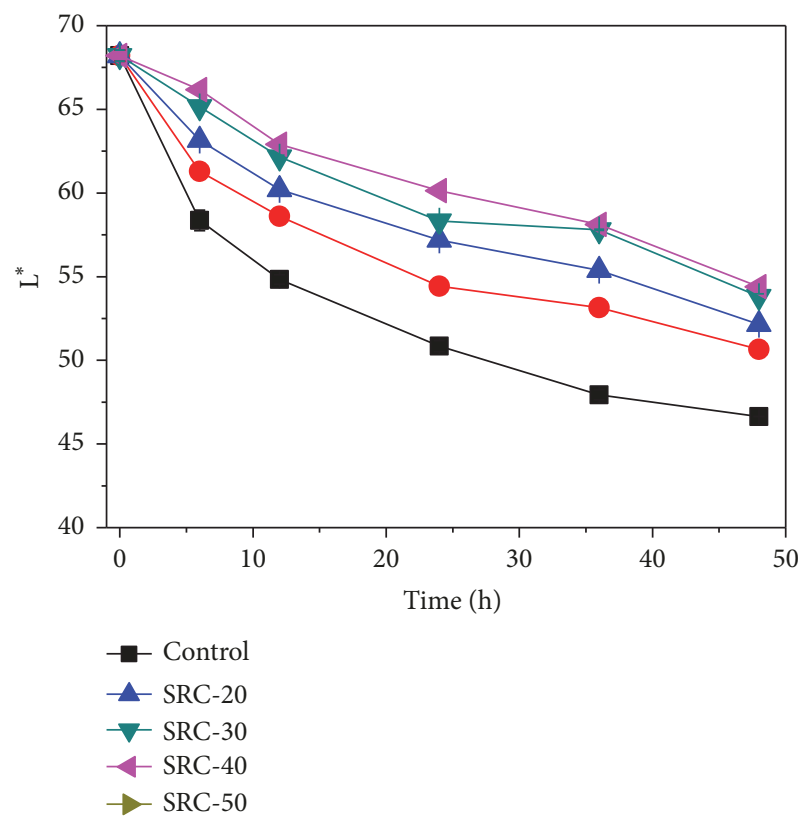

(a)

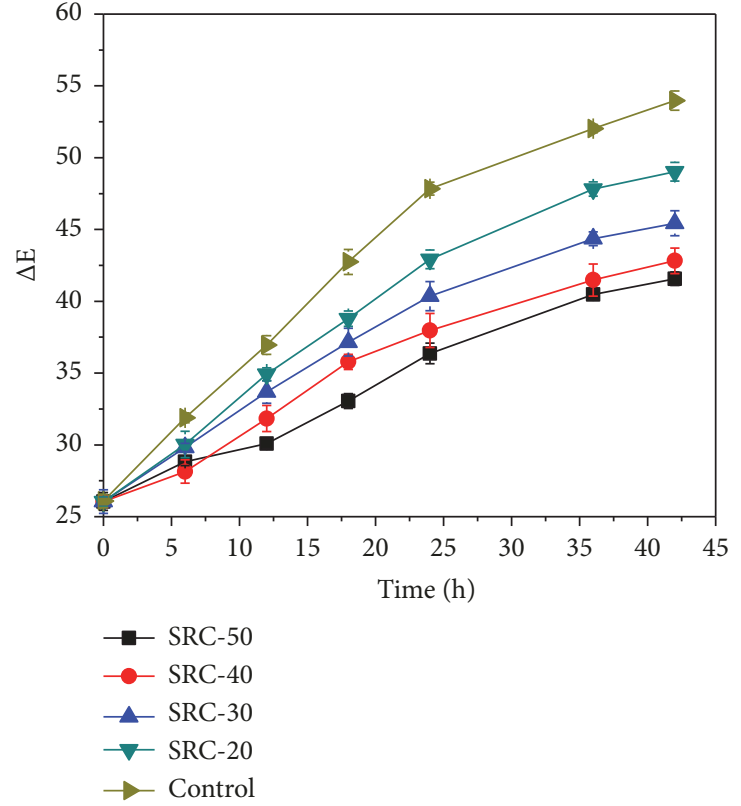

(b)

FIGURE 8: The changes of the color difference of the fresh cut potatoes coated with the SRC microparticles.

$\mathrm{NaIO}_{4}$ concentrations, which was beneficial in introducing more sulphurous acid groups. Moreover, the SRC dispersions could form the free-standing cellulose films after natural drying, which exhibited the higher tensile stress at the same strain $(<0.8 \%)$ with the increase of oxidation and grafting. Meanwhile, the SRC dispersions had enhanced antioxidant activity with the increasing solid contents or high oxidation and grafting degrees. Eventually, the color changes measurement of fresh-cut photo slices treated with SRC diapersions confirmed that the SRC effectively inhibited the browning of potato slices, which could be a good candidate for using as biodegradable coating material for food preservation. 


\section{Data Availability}

No data were used to support this study.

\section{Conflicts of Interest}

The authors declare no competing financial interest.

\section{Acknowledgments}

This work was supported by the project of the Fundamental Research Funds for the Central Universities (2662018PY060).

\section{References}

[1] J. Khourya, I. Seleznevab, S. Pestovc et al., "Surface bioactivation of PEEK by neutral atom beam technology," Bioactive Materials, vol. 4, pp. 132-141, 2019.

[2] Z. H. Chen, Y. J. Jin, W. Z. Yang et al., "Fabrication and characterization of polypyrrole coatings by embedding antimony modified $\mathrm{SnO} 2$ nanoparticles," Journal of Industrial and Engineering Chemistry, vol. 75, no. 25, pp. 178-186, 2019.

[3] H. Wang, D. Di, Y. Zhao, R. Yuan, and Y. Zhu, "A multifunctional polymer composite coating assisted with poreforming agent: Preparation, superhydrophobicity and corrosion resistance," Progress in Organic Coatings, vol. 75, no. 25, pp. 178186, 2019.

[4] H. Bai, C. Huang, H. Xiu et al., "Significantly improving oxygen barrier properties of polylactide via constructing parallelaligned shish-kebab-like crystals with well-interlocked boundaries," Biomacromolecules, vol. 15, no. 4, pp. 1507-1514, 2014.

[5] M. A. Osman, V. Mittal, M. Morbidelli, and U. W. Suter, "Epoxylayered silicate nanocomposites and their gas permeation properties," Macromolecules, vol. 37, no. 19, pp. 7250-7257, 2004.

[6] X. Zhang, Z. Liu, Y. Li et al., "Robust superhydrophobic epoxy composite coating prepared by dual interfacial enhancement," Chemical Engineering Journal, vol. 371, no. 1, pp. 276-285, 2019.

[7] Y. G. Zhang, S. F. Zhang, and S. 1. Wu, "Room-temperature fabrication of TiO2-PHEA nanocomposite coating with high transmittance and durable superhydrophilicity," Chemical Engineering Journal, vol. 371, no. 1, pp. 609-617, 2019.

[8] H. Seok, A. Ali, J. H. Seo et al., "ZnO:Ga-graded ITO electrodes to control interface between PCBM and ITO in planar perovskite solar cells," Science and Technology of Advanced Materials, vol. 20, no. 1, pp. 389-400, 2019.

[9] Z. Wang, S. Zhao, W. Zhang, C. Qi, S. Zhang, and J. Li, "Bioinspired cellulose nanofiber-reinforced soy protein resin adhesives with dopamine-induced codeposition of "water-resistant" interphases," Applied Surface Science, vol. 478, pp. 441-450, 2019.

[10] P. J. Espitia, C. A. Fuenmayor, and C. G. Otoni, "Nanoemulsions: synthesis, characterization, and application in bio-based active food packaging," Comprehensive Reviews in Food Science and Food Safety, vol. 18, no. 1, pp. 264-285, 2019.

[11] Z. J. Lia, X. H. Yanga, W. Li, and H. B. Liu, "Stimuli-responsive cellulose paper materials," Carbohydrate Polymers, vol. 210, no. 15, pp. 264-285, 2019.

[12] G. Sun, W. Chi, C. Zhang, S. Xu, J. Li, and L. Wang, "Developing a green film with $\mathrm{pH}$-sensitivity and antioxidant activity based on к-carrageenan and hydroxypropyl methylcellulose incorporating Prunus maackii juice," Food Hydrocolloids, vol. 94, pp. 345-353, 2019.

[13] N. F. Bakry, M. I. N. Isa, and N. M. Sarbon, "Effect of sorbitol at different concentrations on the functional properties of gelatin/carboxymethyl cellulose (CMC)/chitosan composite films," International Food Research Journal, vol. 24, no. 4, pp. 1753-1762, 2017.

[14] M. Gällstedt and M. S. Hedenqvist, "Packaging-related mechanical and barrier properties of pulp-fiber-chitosan sheets," Carbohydrate Polymers, vol. 63, no. 1, pp. 46-53, 2006.

[15] K. Lokamatha Swamy, B. Satyanath, S. Shantakumar, D. Manjula, H. Mohammedi, and A. Farhana, "Matrix embedded microspherules containing indomethacin as controlled drug delivery systems," Current Drug Delivery, vol. 5, no. 4, pp. 248255, 2008.

[16] F. Karasu, L. Müller, H. Ridaoui et al., "Organic-inorganic hybrid planarization and water vapor barrier coatings on cellulose nanofibrils substrates," Frontiers in Chemistry, vol. 6, article no. 571, 2018.

[17] M. Wu, C. Zhang, J. Pi, C. Liu, J. Yang, and Z. Xu, "Cellulose nanocrystals as anti-oil nanomaterials for separating crude oil from aqueous emulsions and mixtures," Journal of Materials Chemistry A, vol. 7, no. 12, pp. 7033-7041, 2019.

[18] O. Nechyporchuk, M. N. Belgacem, and J. Bras, "Production of cellulose nanofibrils: a review of recent advances," Industrial Crops and Products, vol. 93, pp. 2-25, 2016.

[19] J. Leitner, B. Hinterstoisser, M. Wastyn, J. Keckes, and W. Gindl, "Sugar beet cellulose nanofibril-reinforced composites," Cellulose, vol. 14, no. 5, pp. 419-425, 2007.

[20] S. Eyley and W. Thielemans, "Surface modification of cellulose nanocrystals," Nanoscale, vol. 6, no. 14, pp. 7764-7779, 2014.

[21] B. Thomas, M. C. Raj, A. K. B et al., "Nanocellulose, a versatile green platform: from biosources to materials and their applications," Chemical Reviews, vol. 118, no. 24, pp. 11575-11625, 2018.

[22] A. Borkowski, T. Cłapa, M. Szala, A. Gąsiński, and M. Selwet, "Synthesis of $\mathrm{SiC} / \mathrm{Ag} /$ cellulose nanocomposite and its antibacterial activity by reactive oxygen species generation," Nanomaterials, vol. 6, no. 9, 2016.

[23] K. Liu, X. X. Lin, L. H. Chen et al., "Preparation of microfibrillated cellulose/chitosan-benzalkonium chloride biocomposite for enhancing antibacterium and strength of sodium alginate films," Journal of Agricultural and Food Chemistry, vol. 61, no. 26, Article ID 693278, 2013.

[24] L. N. Nthunya, M. L. Masheane, S. P. Malinga et al., "Greener approach to prepare electrospun antibacterial $\beta$ cyclodextrin/cellulose acetate nanofibers for removal of bacteria from water," ACS Sustainable Chemistry \& Engineering, vol. 5, no. 1, pp. 153-160, 2017.

[25] H. Arnon-Rips, R. Porat, and E. Poverenov, "Enhancement of agricultural produce quality and storability using citralbased edible coatings; the valuable effect of nano-emulsification in a solid-state delivery on fresh-cut melons model," Food Chemistry, vol. 277, pp. 205-212, 2019.

[26] S. Shah, M. Jahangir, M. Qaisar et al., "Storage stability of kinnow fruit (Citrus reticulata) as affected by CMC and guar gum-based silver nanoparticle coatings," Molecules, vol. 20, no. 12, pp. 22645-22661, 2015.

[27] Y. Zhu, X. Luo, X. Wu et al., "Cellulose gel dispersions: fascinating green particles for the stabilization of oil/water Pickering emulsion," Cellulose, vol. 24, no. 1, pp. 207-217, 2017. 
[28] S. Liu, Y. Zhu, Y. Wu, A. Lue, and C. Zhang, "Hydrophobic modification of regenerated cellulose microparticles with enhanced emulsifying capacity for O/W Pickering emulsion," Cellulose, vol. 26, no. 10, pp. 6215-6228, 2019.

[29] Q. Zhang, D. Lin, and S. Yao, "Review on biomedical and bioengineering applications of cellulose sulfate," Carbohydrate Polymers, vol. 132, pp. 311-322, 2015.

[30] J. Liu, Q. Xu, J. Zhang et al., "Preparation, composition analysis and antioxidant activities of konjac oligo-glucomannan," Carbohydrate Polymers, vol. 130, pp. 398-404, 2015.

[31] X. Liu, Q. Yang, Y. Lu et al., "Effect of purslane (Portulaca oleracea L.) extract on anti-browning of fresh-cut potato slices during storage," Food Chemistry, vol. 283, pp. 445-453, 2019. 


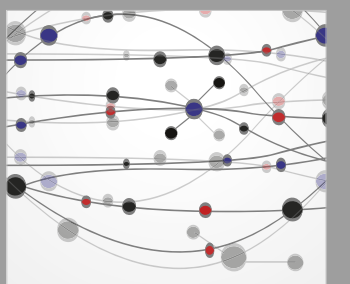

The Scientific World Journal
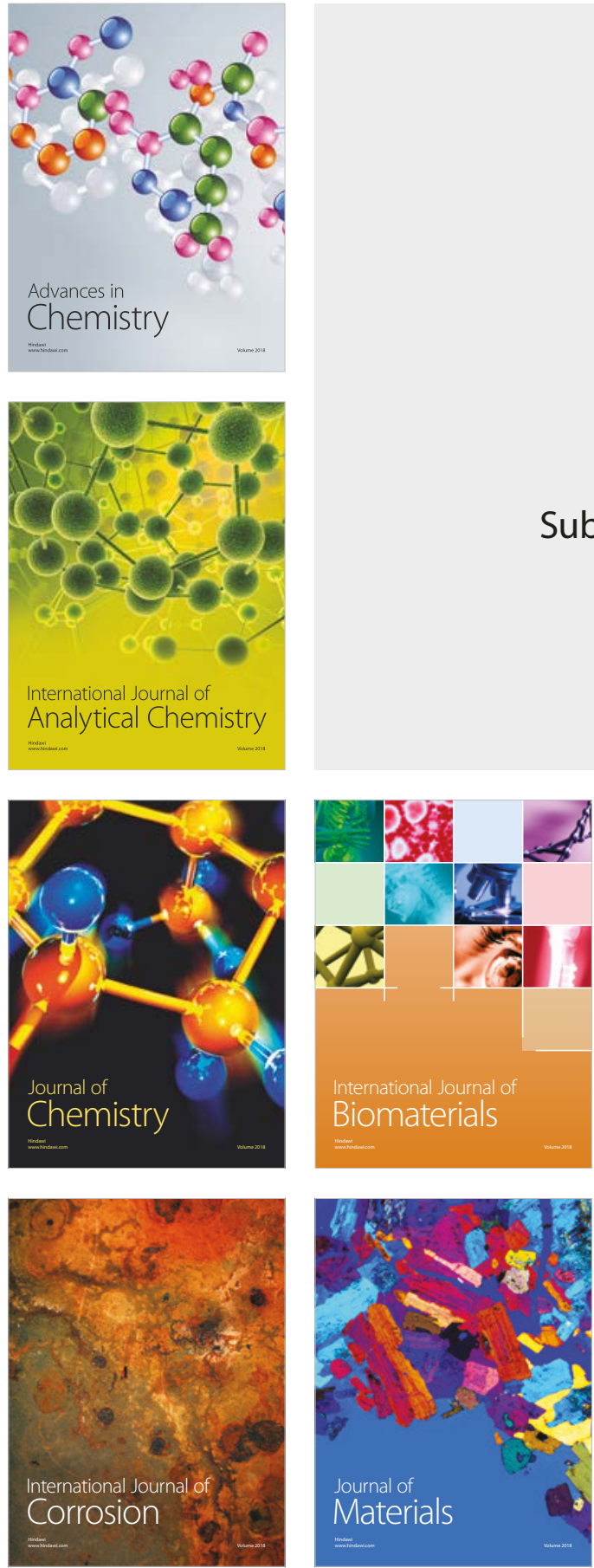

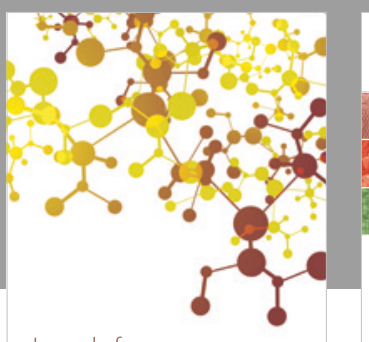

Journal of

Applied Chemistry
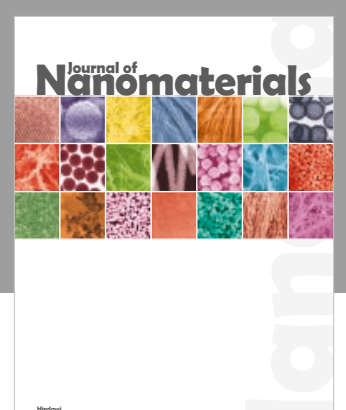

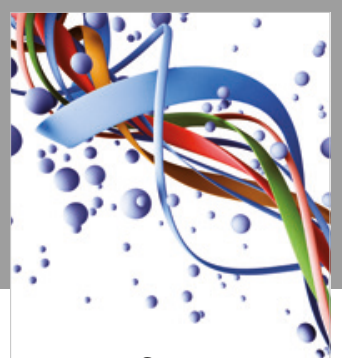

Scientifica

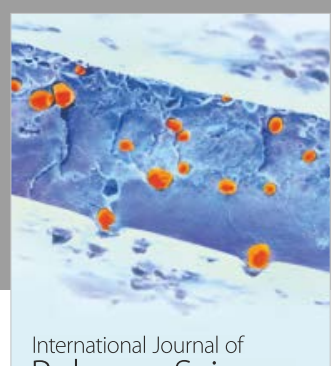

Polymer Science

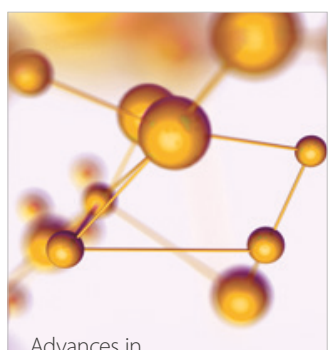

Physical Chemistry
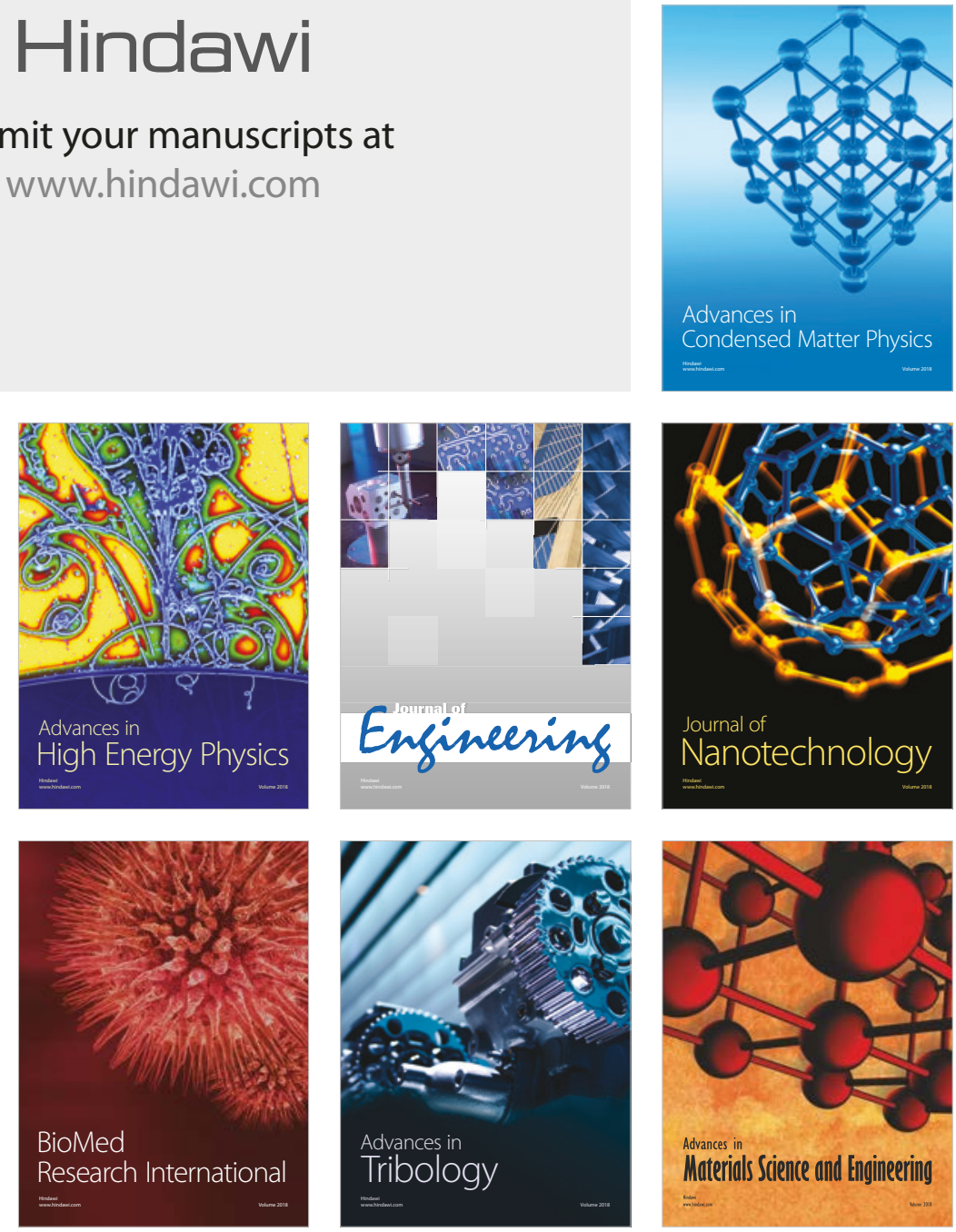